\title{
Approximation Property of the Stationary Stokes Equations with the Periodic Boundary Condition
}

\author{
Soon-Mo Jung $\mathbb{D}^{1}$ and Jaiok Roh $\mathbb{D}^{2}$ \\ ${ }^{1}$ Mathematics Section, College of Science and Technology, Hongik University, 30016 Sejong, Republic of Korea \\ ${ }^{2}$ Department of Finance and Information Statistics, Institute of Statistics, Hallym University, Chuncheon, \\ Kangwon-Do 24252, Republic of Korea
}

Correspondence should be addressed to Jaiok Roh; joroh@hallym.ac.kr

Received 29 June 2018; Accepted 12 September 2018; Published 9 October 2018

Academic Editor: Mark A. McKibben

Copyright (c) 2018 Soon-Mo Jung and Jaiok Roh. This is an open access article distributed under the Creative Commons Attribution License, which permits unrestricted use, distribution, and reproduction in any medium, provided the original work is properly cited.

In this paper, we will consider the stationary Stokes equations with the periodic boundary condition and we will study approximation property of the solutions by using the properties of the Fourier series. Finally, we will discuss that our estimation for approximate solutions is optimal.

\section{Introduction}

The study of stability problems for various functional equations originated from a famous talk presented by Ulam in 1940. In this talk, he discussed a problem concerning the stability of homomorphisms. And Obłoza [1, 2] first investigated the Hyers-Ulam stability of the linear differential equations which have the form $y^{\prime}(x)+g(x) y(x)=r(x)$. Thereafter, a number of mathematicians have dealt with this subject for different types of differential equations (see [3-8]).

For an open interval $I=(a, b)$ of $\mathbb{R}$ with $-\infty \leq a<b \leq$ $+\infty$, we consider the linear differential equation of $n$th order

$$
\mathscr{F}\left(y^{(n)}, y^{(n-1)}, \ldots, y^{\prime}, y, x\right)=0
$$

defined on $I$, where $y: I \longrightarrow \mathbb{C}$ is an $n$ times continuously differentiable function.

We say that the differential equation (1) satisfies the HyersUlam stability provided the following statement is true for any $\varepsilon>0$ : if an $n$ times continuously differentiable function $y$ : $I \longrightarrow \mathbb{C}$ satisfies the differential inequality

$$
\left|\mathscr{F}\left(y^{(n)}, y^{(n-1)}, \ldots, y^{\prime}, y, x\right)\right| \leq \varepsilon
$$

for all $x \in I$, then there exists a solution $y_{0}: I \longrightarrow \mathbb{C}$ to (1) such that

$$
\left|y(x)-y_{0}(x)\right| \leq K(\varepsilon)
$$

for all $x \in I$, where $K(\varepsilon)$ depends on $\varepsilon$ only and satisfies $\lim _{\varepsilon \rightarrow 0} K(\varepsilon)=0$.

Recently, several mathematicians investigated the HyersUlam stability for the partial differential equations. One can refer to [9-14].

In this paper, we will investigate approximate properties of the solutions for the stationary Stokes equations with the periodic boundary condition. The stationary Stokes problem associated with the space periodicity condition is the following one: For a given $\mathbf{f}$, find $\mathbf{u}$ and $p$ such that

$$
\begin{aligned}
-\Delta \mathbf{u}+\nabla p & =\mathbf{f} \quad \text { in } Q \\
\nabla \cdot \mathbf{u} & =0 \quad \text { in } Q \\
\mathbf{u}\left(x+L e_{i}\right) & =\mathbf{u}(x) \quad \forall x \in \mathbb{R}^{n},
\end{aligned}
$$

where $\left\{e_{1}, \ldots, e_{n}\right\}$ is the canonical basis of $\mathbb{R}^{n}, L$ is the period in the $i$-th direction, and $Q=(0, L)^{n}$ is the cube of the period.

The advantage of the boundary condition (6) is that it leads to a simple functional setting, while many of the mathematical difficulties remain unchanged. In fact, in the next section we will introduce in detail the corresponding functional setting of the problem.

Finally, we will discuss that our estimation for approximate solutions is optimal. 


\section{Preliminary Results}

In this section, we will introduce the useful functional settings and preliminary results for the solutions of the stationary Stokes equations with the periodic boundary condition. For the materials of this section, we totally refer to the book by Roger Temam [15]. So if the reader wants to understand more deeply, one can refer to this book.

For the functional spaces of the solutions, we will consider the Lebesque space $L^{2}\left(\mathbb{R}^{n}\right)$ with the periodic boundary condition. We set by $H^{m}(\Omega)$ the Sobolev space of functions which are in $L^{2}(\Omega)$, with all their derivatives of order $\leq m$. Then, $H^{m}(\Omega)$ is a Hilbert space with the inner product and the norm

$$
\begin{aligned}
(\mathbf{u}, \mathbf{v})_{m} & =\sum_{[\alpha] \leq m}\left(D^{\alpha} \mathbf{u}, D^{\alpha} \mathbf{v}\right), \\
|\mathbf{u}|_{m} & =\left[(\mathbf{u}, \mathbf{u})_{m}\right]^{1 / 2},
\end{aligned}
$$

where $\alpha=\left(\alpha_{1}, \ldots, \alpha_{n}\right), \alpha_{i} \in \mathbb{N}_{0},[\alpha]=\alpha_{1}+\cdots+\alpha_{n}$, and

$$
D^{\alpha}=D_{1}^{\alpha_{1}} \cdots D_{n}^{\alpha_{n}}=\frac{\partial^{[\alpha]}}{\partial x_{1}^{\alpha_{1}} \cdots \partial x_{n}^{\alpha_{n}}} .
$$

We also set by $H_{p}^{m}(Q), m \in \mathbb{N}_{0}$, the space of functions which are periodic with period $Q$ :

$$
\mathbf{u}\left(\mathbf{x}+L \mathbf{e}_{i}\right)=\mathbf{u}(\mathbf{x}) \quad \forall i=1, \ldots, n .
$$

For $m=0, H_{p}^{0}(Q)$ means simply $L^{2}(Q)$. Then, for an arbitrary $m \in \mathbb{N}, H_{p}^{m}(Q)$ is a Hilbert space with the inner product

$$
(\mathbf{u}, \mathbf{v})_{m}=\sum_{[\alpha] \leq m} \int_{Q} D^{\alpha} \mathbf{u}(\mathbf{x}) D^{\alpha} \mathbf{v}(\mathbf{x}) d \mathbf{x} .
$$

And the functions in $H_{p}^{m}(Q)$ are characterized by their Fourier series expansion

$$
\begin{gathered}
H_{p}^{m}(Q)=\left\{\mathbf{u}: \mathbf{u}=\sum_{\mathbf{k} \in \mathbb{Z}^{n}} c_{\mathbf{k}} e^{2 i \pi \mathbf{k} \cdot \mathbf{x} / L}, \bar{c}_{\mathbf{k}}\right. \\
\left.=c_{-\mathbf{k}}, \sum_{\mathbf{k} \in \mathbb{Z}^{n}}|\mathbf{k}|^{2 m}\left|\mathcal{c}_{\mathbf{k}}\right|^{2}<\infty\right\} .
\end{gathered}
$$

We also denote

$$
\dot{H}_{p}^{m}(Q)=\left\{\mathbf{u} \in H_{p}^{m}(Q) \text { of type }(11): c_{0}=0\right\} .
$$

Then, for $m \in \mathbb{N}, \dot{H}_{p}^{m}(Q)$ is a Hilbert space for the norm $\left[\sum_{\mathbf{k} \in \mathbb{Z}^{n}}|\mathbf{k}|^{2 m}\left|\mathcal{c}_{\mathbf{k}}\right|^{2}\right]^{1 / 2}$, and $\dot{H}_{p}^{m}(Q)$ and $\dot{H}_{p}^{-m}(Q)$ are in duality for all $m \in \mathbb{N}$.

Now, we introduce two important function spaces,

$$
\begin{aligned}
& \mathbf{V}=\left\{\mathbf{u} \in \mathbf{H}_{p}^{1}(Q): \nabla \cdot \mathbf{u}=0 \text { in } \mathbb{R}^{n}\right\}, \\
& \mathbf{H}=\left\{\mathbf{u} \in \mathbf{H}_{p}^{0}(Q): \nabla \cdot \mathbf{u}=0 \text { in } \mathbb{R}^{n}\right\},
\end{aligned}
$$

where $\mathbf{H}_{p}^{m}(Q)=\left\{H_{p}^{m}(Q)\right\}^{n}$. We also introduce the inner product and the norm

$$
\begin{aligned}
((\mathbf{u}, \mathbf{v})) & =\sum_{i=1}^{n}\left(\frac{\partial \mathbf{u}}{\partial x_{i}}, \frac{\partial \mathbf{v}}{\partial x_{i}}\right), \\
\|\mathbf{u}\| & =\{((\mathbf{u}, \mathbf{u}))\}^{1 / 2}
\end{aligned}
$$

One notes that $\mathbf{V}$ is a Hilbert space with this norm. Also, the dual $\mathbf{V}^{\prime}$ of $\mathbf{V}$ is

$$
\mathbf{V}^{\prime}=\left\{\mathbf{u} \in \mathbf{H}_{p}^{-1}(Q)=\left(\mathbf{H}_{p}^{1}(Q)\right)^{\prime}: \nabla \cdot \mathbf{u}=0 \text { in } \mathbb{R}^{n}\right\} ;
$$

$\|\cdot\|_{\mathbf{V}^{\prime}}$ will denote the dual norm of $\|\cdot\|$ on $\mathbf{V}^{\prime}$. For the boundary value, due to trace theorem we have that $\mathbf{u} \in \mathbf{V}$ if and only if its restriction $\left.\mathbf{u}\right|_{Q}$ to $Q$ belongs to

$$
\left\{\mathbf{v} \in \mathbf{H}^{1}(Q): \nabla \cdot \mathbf{v}=0,\left.\quad \mathbf{v}\right|_{\Gamma_{j+n}}=\left.\mathbf{v}\right|_{\Gamma_{j}} j=1, \ldots, n\right\}
$$

where we have numbered the faces $\Gamma_{1}, \ldots, \Gamma_{2 n}$ of $Q$ as follows:

$$
\begin{gathered}
\Gamma_{j}=\partial Q \cap\left\{x_{j}=0\right\}, \\
\Gamma_{j+n}=\partial Q \cap\left\{x_{j}=L\right\},
\end{gathered}
$$

and $\left.\mathbf{v}\right|_{\Gamma_{i}}$ is an improper notation for the trace of $\mathbf{v}$ on $\Gamma_{i}$. And $\mathbf{u} \in \mathbf{H}$ if and only if $\mathbf{u}$ belongs to

$$
\begin{aligned}
\{\mathbf{v} & \in \mathbf{L}^{2}(Q): \nabla \cdot \mathbf{v}=0,\left.\mathbf{v} \cdot \nu\right|_{\Gamma_{j+n}}=-\left.\mathbf{v} \cdot \nu\right|_{\Gamma_{j}} j \\
& =1, \ldots, n\} .
\end{aligned}
$$

Now, let us look at the stationary Stokes problem (4) with the periodic boundary condition (6); given $\mathbf{f} \in \dot{\mathbf{H}}_{p}^{0}(Q)$ or $\dot{\mathbf{H}}_{p}^{-1}(Q)$, find $\mathbf{u} \in \dot{\mathbf{H}}_{p}^{1}(Q)$ and $p \in L^{2}(Q)$ such that

$$
\begin{aligned}
-\Delta \mathbf{u}+\nabla p=\mathbf{f} & \text { in } Q \\
\nabla \cdot \mathbf{u}=0 & \text { in } Q .
\end{aligned}
$$

Here, to solve the above problem we use the Fourier series. Let us introduce the Fourier expansions of $\mathbf{u}, p$, and f;

$$
\begin{aligned}
& \mathbf{u}=\sum_{\mathbf{k} \in \mathbb{Z}^{n}} u_{\mathbf{k}} e^{2 \pi i \mathbf{k} \cdot \mathbf{x} / L}, \\
& p=\sum_{\mathbf{k} \in \mathbb{Z}^{n}} p_{\mathbf{k}} e^{2 \pi i \mathbf{k} \cdot \mathbf{x} / L}, \\
& \mathbf{f}=\sum_{\mathbf{k} \in \mathbb{Z}^{n}} f_{\mathbf{k}} e^{2 \pi i \mathbf{k} \cdot \mathbf{x} / L} .
\end{aligned}
$$

Equation (20) reduces for every $\mathbf{k} \neq 0$ to

$$
\frac{4 \pi^{2}|\mathbf{k}|^{2}}{L^{2}} u_{\mathbf{k}}+\frac{2 \pi i \mathbf{k}}{L} p_{\mathbf{k}}=f_{\mathbf{k}}
$$

and

$$
\mathbf{k} \cdot u_{\mathbf{k}}=0 \text {. }
$$


Taking the scalar product of (22) with $\mathbf{k}$ and using (23) we find the $p_{\mathbf{k}}$ 's:

$$
p_{\mathbf{k}}=\frac{L \mathbf{k} \cdot f_{\mathbf{k}}}{2 \pi i|\mathbf{k}|^{2}} \quad \text { for } \mathbf{k} \in \mathbb{Z}^{n}, \mathbf{k} \neq 0
$$

then (22) provided the $u_{\mathbf{k}}$ 's;

$$
u_{\mathbf{k}}=\frac{L^{2}}{4 \pi^{2}|\mathbf{k}|^{2}}\left(f_{\mathbf{k}}-\frac{\left(\mathbf{k} \cdot f_{\mathbf{k}}\right) \mathbf{k}}{|\mathbf{k}|^{2}}\right)
$$

for $\mathbf{k} \in \mathbb{Z}^{n}, \mathbf{k} \neq 0$.

By definition (11) of $\mathbf{H}_{p}^{m}(Q)$, if $\mathbf{f} \in \dot{\mathbf{H}}_{p}^{0}(Q)$ then $\mathbf{u} \in \dot{\mathbf{H}}_{p}^{2}(Q)$ and $p \in \dot{\mathbf{H}}_{p}^{1}(Q)$; if $\mathbf{f} \in \dot{\mathbf{H}}_{p}^{-1}(Q)$ then $u \in \dot{\mathbf{H}}_{p}^{1}(Q)$ and $p \in \dot{\mathbf{H}}_{p}^{0}(Q)$. Now if $\mathbf{f}$ belongs to $\mathbf{H}$, then $\mathbf{k} \cdot f_{\mathbf{k}}=0$ for every $\mathbf{k}$ so that $p=0$ and $u_{\mathbf{k}}=f_{\mathbf{k}} L^{2} / 4 \pi^{2}|\mathbf{k}|^{2}$.

\section{Approximate Properties for the Solutions}

In this section, we will discuss approximate properties for the solutions of the stationary Stokes equations with the periodic boundary condition. In this paper, we will prove theorems for $n=2$ while one can extend our result to $\mathbb{R}^{n}$.

Theorem 1. Let the functions $\mathbf{v} \in \dot{\mathbf{H}}_{p}^{2}(Q)$ and $q \in \dot{\mathbf{H}}_{p}^{1}(Q)$ satisfy the equations

$$
\begin{aligned}
-\Delta \mathbf{v}+\nabla q-\mathbf{f}=\mathbf{g} & \text { in } Q \\
\nabla \cdot \mathbf{v}=0 & \text { in } Q
\end{aligned}
$$

where $\|\mathbf{g}\|_{L^{2}} \leq \varepsilon$ and $\mathbf{f}, \mathbf{g} \in \dot{\mathbf{H}}_{p}^{0}(Q)$. Then there exist $\mathbf{u} \in \dot{\mathbf{H}}_{p}^{2}(Q)$ and $p \in \dot{\mathbf{H}}_{p}^{1}(\mathrm{Q})$ satisfying

$$
\begin{aligned}
-\Delta \mathbf{u}+\nabla p-\mathbf{f}=0 & \text { in } Q \\
\nabla \cdot \mathbf{u}=0 & \text { in } Q
\end{aligned}
$$

such that

$$
\begin{gathered}
\|\mathbf{u}-\mathbf{v}\|_{H^{i}} \leq K_{i}\|\mathbf{g}\|_{L^{2}} \leq K_{i} \varepsilon \quad \text { for } i=0,1,2, \\
\|p-q\|_{H^{i}} \leq M_{i}\|\mathbf{g}\|_{L^{2}} \leq M_{i} \varepsilon \quad \text { for } i=0,1
\end{gathered}
$$

for some constants $K_{i}$ and $M_{i}$.

Proof. For existence of the solutions $\mathbf{u} \in \dot{\mathbf{H}}_{p}^{2}(Q)$ and $p \in$ $\dot{\mathbf{H}}_{p}^{1}(Q)$, One can prove by (24) and (25). Next, to obtain (28) and (29) we denote the Fourier expansions of $\mathbf{v}, q$, and $\mathbf{g}$ as the following;

$$
\begin{aligned}
& \mathbf{v}=\sum_{\mathbf{k} \in \mathbb{Z}^{2}} v_{\mathbf{k}} e^{2 \pi i \mathbf{k} \cdot \mathbf{x} / L}, \\
& q=\sum_{\mathbf{k} \in \mathbb{Z}^{2}} q_{\mathbf{k}} e^{2 \pi i \mathbf{k} \cdot \mathbf{x} / L}, \\
& \mathbf{g}=\sum_{\mathbf{k} \in \mathbb{Z}^{2}} g_{\mathbf{k}} e^{2 \pi i \mathbf{k} \cdot \mathbf{x} / L} .
\end{aligned}
$$

Then, by (24), (25), and (26) we obtain

$$
q_{\mathbf{k}}=\frac{L \mathbf{k} \cdot\left(f_{\mathbf{k}}+g_{\mathbf{k}}\right)}{2 \pi i|\mathbf{k}|^{2}} \quad \text { for } \mathbf{k} \in \mathbb{Z}^{2}, \mathbf{k} \neq 0
$$

and

$$
\begin{array}{r}
v_{\mathbf{k}}=\frac{L^{2}}{4 \pi^{2}|\mathbf{k}|^{2}}\left(\left(f_{\mathbf{k}}+g_{\mathbf{k}}\right)-\frac{\left(\mathbf{k} \cdot\left[f_{\mathbf{k}}+g_{\mathbf{k}}\right]\right) \mathbf{k}}{|\mathbf{k}|^{2}}\right) \\
\text { for } \mathbf{k} \in \mathbb{Z}^{2}, \mathbf{k} \neq 0 .
\end{array}
$$

Also, by (24), (25), and (27) we obtain

$$
p_{\mathbf{k}}=\frac{L \mathbf{k} \cdot f_{\mathbf{k}}}{2 \pi i|\mathbf{k}|^{2}} \quad \text { for } \mathbf{k} \in \mathbb{Z}^{2}, \mathbf{k} \neq 0
$$

and

$$
u_{\mathbf{k}}=\frac{L^{2}}{4 \pi^{2}|\mathbf{k}|^{2}}\left(f_{\mathbf{k}}-\frac{\left(\mathbf{k} \cdot f_{\mathbf{k}}\right) \mathbf{k}}{|\mathbf{k}|^{2}}\right)
$$

for $\mathbf{k} \in \mathbb{Z}^{2}, \mathbf{k} \neq 0$.

So, by (31)-(34), for the Fourier expansions of $\mathbf{u}-\mathbf{v}$ and $p-q$ we have

$$
\begin{aligned}
& u_{\mathbf{k}}-v_{\mathbf{k}}=-\frac{L^{2}}{4 \pi^{2}|\mathbf{k}|^{2}}\left(g_{\mathbf{k}}-\frac{\left(\mathbf{k} \cdot g_{\mathbf{k}}\right) \mathbf{k}}{|\mathbf{k}|^{2}}\right), \\
& p_{\mathbf{k}}-q_{\mathbf{k}}=-\frac{L \mathbf{k} \cdot g_{\mathbf{k}}}{2 \pi i|\mathbf{k}|^{2}} .
\end{aligned}
$$

Then, for $|\mathbf{k}| \geq 2$, from (35) we have

$$
\left|u_{\mathbf{k}}-v_{\mathbf{k}}\right| \leq \frac{L^{2}}{8 \pi^{2}}\left|g_{\mathbf{k}}\right|
$$

and for $|\mathbf{k}|=1$ and $g_{\mathbf{k}}=\left(g_{\mathbf{k}}^{1}, g_{\mathbf{k}}^{2}\right)$, we have

$$
\begin{aligned}
& g_{\mathbf{k}}-\frac{\left(\mathbf{k} \cdot g_{\mathbf{k}}\right) \mathbf{k}}{|\mathbf{k}|^{2}}=\left(0, g_{\mathbf{k}}^{2}\right), \quad \text { for } \mathbf{k}=(1,0), \\
& g_{\mathbf{k}}-\frac{\left(\mathbf{k} \cdot g_{\mathbf{k}}\right) \mathbf{k}}{|\mathbf{k}|^{2}}=\left(0, g_{\mathbf{k}}^{2}\right), \quad \text { for } \mathbf{k}=(-1,0), \\
& g_{\mathbf{k}}-\frac{\left(\mathbf{k} \cdot g_{\mathbf{k}}\right) \mathbf{k}}{|\mathbf{k}|^{2}}=\left(g_{\mathbf{k}}^{1}, 0\right), \quad \text { for } \mathbf{k}=(0,1), \\
& g_{\mathbf{k}}-\frac{\left(\mathbf{k} \cdot g_{\mathbf{k}}\right) \mathbf{k}}{|\mathbf{k}|^{2}}=\left(g_{\mathbf{k}}^{1}, 0\right), \quad \text { for } \mathbf{k}=(0,-1)
\end{aligned}
$$

which implies

$$
\left|u_{\mathbf{k}}-v_{\mathbf{k}}\right| \leq \frac{L^{2}}{4 \pi^{2}}\left|g_{\mathbf{k}}\right|
$$

Hence, we have

$$
\|\mathbf{u}-\mathbf{v}\|_{L^{2}}=\left[\sum_{\mathbf{k} \in \mathbb{Z}^{n}}\left|u_{\mathbf{k}}-v_{\mathbf{k}}\right|^{2}\right]^{1 / 2} \leq \frac{L^{2}}{4 \pi^{2}}\|\mathbf{g}\|_{L^{2}} \leq K_{1} \varepsilon
$$


and

$$
\|p-q\|_{L^{2}}=\left[\sum_{\mathbf{k} \in \mathbb{Z}^{n}}\left|p_{\mathbf{k}}-q_{\mathbf{k}}\right|^{2}\right]^{1 / 2} \leq \frac{L}{2 \pi}\|\mathbf{g}\|_{L^{2}} \leq M_{1} \varepsilon .
$$

Similarly, for $H^{1}$-norm of $\mathbf{u}-\mathbf{v}$ and $p-q$, we obtain

$$
\begin{aligned}
\|\mathbf{u}-\mathbf{v}\|_{H^{1}} & =\left[\sum_{\mathbf{k} \in \mathbb{Z}^{n}}|\mathbf{k}|^{2}\left|u_{\mathbf{k}}-v_{\mathbf{k}}\right|^{2}\right]^{1 / 2} \leq \frac{L^{2}}{4 \pi^{2}}\|\mathbf{g}\|_{L^{2}} \\
& \leq K_{2} \varepsilon
\end{aligned}
$$

and

$$
\begin{aligned}
\|p-q\|_{H^{1}} & =\left[\sum_{\mathbf{k} \in \mathbb{Z}^{n}}|\mathbf{k}|^{2}\left|p_{\mathbf{k}}-q_{\mathbf{k}}\right|^{2}\right]^{1 / 2} \leq \frac{L}{2 \pi}\|\mathbf{g}\|_{L^{2}} \\
& \leq M_{2} \varepsilon .
\end{aligned}
$$

Also, for $\mathrm{H}^{2}$-norm of $\mathbf{u}-\mathbf{v}$, we have

$$
\begin{aligned}
\|\mathbf{u}-\mathbf{v}\|_{H^{2}} & =\left[\sum_{\mathbf{k} \in \mathbb{Z}^{n}}|\mathbf{k}|^{4}\left|u_{\mathbf{k}}-v_{\mathbf{k}}\right|^{2}\right]^{1 / 2} \leq \frac{L^{2}}{4 \pi^{2}}\|\mathbf{g}\|_{L^{2}} \\
& \leq K_{3} \varepsilon .
\end{aligned}
$$

Therefore, by (39)-(43), we complete the proof.

Remark 2. We consider the function $\mathbf{g}$ as $g_{\mathbf{k}}=(0,0)$ for $\mathbf{k} \neq$ $(1,0)$ and $g_{\mathbf{k}}=(0,1)$ for $\mathbf{k}=(1,0)$. Then, we have

$$
\begin{aligned}
& \|\mathbf{u}-\mathbf{v}\|_{L^{2}}=\left[\sum_{\mathbf{k} \in \mathbb{Z}^{2}}\left|u_{\mathbf{k}}-v_{\mathbf{k}}\right|^{2}\right]^{1 / 2}=\frac{L^{2}}{4 \pi^{2}}\|\mathbf{g}\|_{L^{2}}, \\
& \|\mathbf{u}-\mathbf{v}\|_{H^{1}}=\left[\sum_{\mathbf{k} \in \mathbb{Z}^{2}}|\mathbf{k}|^{2}\left|u_{\mathbf{k}}-v_{\mathbf{k}}\right|^{2}\right]^{1 / 2}=\frac{L^{2}}{4 \pi^{2}}\|\mathbf{g}\|_{L^{2}}, \\
& \|\mathbf{u}-\mathbf{v}\|_{H^{2}}=\left[\sum_{\mathbf{k} \in \mathbb{Z}^{2}}|\mathbf{k}|^{4}\left|u_{\mathbf{k}}-v_{\mathbf{k}}\right|^{2}\right]^{1 / 2}=\frac{L^{2}}{4 \pi^{2}}\|\mathbf{g}\|_{L^{2}} .
\end{aligned}
$$

And we consider the function $\mathbf{g}$ as $g_{\mathbf{k}}=(0,0)$ for $\mathbf{k} \neq(1,0)$ and $g_{\mathbf{k}}=(1,0)$ for $\mathbf{k}=(1,0)$. Then we have

$$
\begin{aligned}
& \|p-q\|_{L^{2}}=\left[\sum_{\mathbf{k} \in \mathbb{Z}^{2}}\left|p_{\mathbf{k}}-q_{\mathbf{k}}\right|^{2}\right]^{1 / 2}=\frac{L}{2 \pi}\|\mathbf{g}\|_{L^{2}}, \\
& \|p-q\|_{H^{1}}=\left[\sum_{\mathbf{k} \in \mathbb{Z}^{2}}|\mathbf{k}|^{2}\left|p_{\mathbf{k}}-q_{\mathbf{k}}\right|^{2}\right]^{1 / 2}=\frac{L}{2 \pi}\|\mathbf{g}\|_{L^{2}} .
\end{aligned}
$$

Hence, our estimation for $K_{i}$ and $M_{i}$ is optimal.

Corollary 3. Let the functions $\mathbf{v} \in \dot{\mathbf{H}}_{p}^{2}(Q)$ and $q \in \dot{\mathbf{H}}_{p}^{1}(Q)$ satisfy the equations

$$
\begin{aligned}
-\Delta \mathbf{v}+\nabla q-\mathbf{f}=\mathbf{g} & \text { in } Q \\
\nabla \cdot \mathbf{v}=0 & \text { in } Q
\end{aligned}
$$

where $\mathbf{f} \in \mathbf{H}$ and $\mathbf{g} \in \dot{\mathbf{H}}_{p}^{0}(Q)$ with $\|\mathbf{g}\|_{L^{2}} \leq \varepsilon$. Then there exist $\mathbf{u} \in \dot{\mathbf{H}}_{p}^{2}(Q)$ and $p \in \dot{\mathbf{H}}_{p}^{1}(Q)$ satisfying

$$
\begin{aligned}
-\Delta \mathbf{u}+\nabla p-\mathbf{f}=0 & \text { in } \mathrm{Q} \\
\nabla \cdot \mathbf{u}=0 & \text { in } \mathrm{Q} .
\end{aligned}
$$

such that

$$
\begin{gathered}
\|\mathbf{u}-\mathbf{v}\|_{H^{i}} \leq K_{i}\|\mathbf{g}\|_{L^{2}} \leq K_{i} \varepsilon \quad \text { for } i=0,1,2, \\
\|p-q\|_{H^{i}} \leq M_{i}\|\mathbf{g}\|_{L^{2}} \leq M_{i} \varepsilon \quad \text { for } i=0,1
\end{gathered}
$$

Corollary 4. Let the functions $\mathbf{v} \in \dot{\mathbf{H}}_{p}^{2}(Q)$ and $q \in \dot{\mathbf{H}}_{p}^{1}(Q)$ satisfy the equations

$$
\begin{aligned}
-\Delta \mathbf{v}+\nabla q-\mathbf{f}=\mathbf{g} & \text { in } Q \\
\nabla \cdot \mathbf{v}=0 & \text { in } Q
\end{aligned}
$$

where $\mathbf{f}, \mathbf{g} \in \mathbf{H}$ with $\|\mathbf{g}\|_{L^{2}} \leq \varepsilon$. Then there exist $\mathbf{u} \in \dot{\mathbf{H}}_{p}^{2}(Q)$ and $p \in \dot{\mathbf{H}}_{p}^{1}(Q)$ satisfying

$$
\begin{aligned}
-\Delta \mathbf{u}+\nabla p-\mathbf{f}=0 & \text { in } Q \\
\nabla \cdot \mathbf{u}=0 & \text { in } Q .
\end{aligned}
$$

such that

$$
\begin{aligned}
& \|\mathbf{u}-\mathbf{v}\|_{H^{i}} \leq K_{i}\|\mathbf{g}\|_{L^{2}} \leq K_{i} \varepsilon \quad \text { for } i=0,1,2, \\
& \|p-q\|_{H^{i}}=0 \quad \text { for } i=0,1 .
\end{aligned}
$$

Corollary 5. Let the functions $\mathbf{v} \in \dot{\mathbf{H}}_{p}^{2}(Q)$ and $q \in \dot{\mathbf{H}}_{p}^{1}(Q)$ satisfy the equations

$$
\begin{aligned}
-\Delta \mathbf{v}+\nabla q-\mathbf{f}=\mathbf{g} & \text { in } Q \\
\nabla \cdot \mathbf{v}=0 & \text { in } Q
\end{aligned}
$$

where $\mathbf{f} \in \dot{\mathbf{H}}_{p}^{0}(Q)$ and $\mathbf{g} \in \mathbf{H}$ with $\|\mathbf{g}\|_{L^{2}} \leq \varepsilon$. Then there exist $\mathbf{u} \in \dot{\mathbf{H}}_{p}^{2}(Q)$ and $p \in \dot{\mathbf{H}}_{p}^{1}(Q)$ satisfying

$$
\begin{aligned}
-\Delta \mathbf{u}+\nabla p-\mathbf{f}=0 & \text { in } Q \\
\nabla \cdot \mathbf{u} & =0 \quad \text { in } Q .
\end{aligned}
$$

such that

$$
\begin{aligned}
& \|\mathbf{u}-\mathbf{v}\|_{H^{i}} \leq K_{i}\|\mathbf{g}\|_{L^{2}} \leq K_{i} \varepsilon \quad \text { for } i=0,1,2, \\
& \|p-q\|_{H^{i}}=0 \quad \text { for } i=0,1 .
\end{aligned}
$$

\section{Data Availability}

No data were used to support this study.

\section{Conflicts of Interest}

The authors declare that they have no conflicts of interest. 


\section{Acknowledgments}

This work was supported by Hallym University Research Fund (HRF-201805-008).

\section{References}

[1] M. Obłoza, "Hyers stability of the linear differential equation," Rocznik Naukowo-Dydaktyczny - Wyższa Szkoła Pedagogiczna im. Komisji Edukacji Narodowej. Prace Matematyczne, no. 13, pp. 259-270, 1993.

[2] M. Obłoza, "Connections between Hyers and Lyapunov stability of the ordinary differential equations," Rocznik Naukowo-Dydaktyczny - Wyższa Szkoła Pedagogiczna im. Komisji Edukacji Narodowej. Prace Matematyczne, no. 14, pp. 141-146, 1997.

[3] S.-M. Jung and S. Min, "Stability of the wave equation with a source," Journal of Function Spaces, vol. 2018, Article ID 8274159, 4 pages, 2018.

[4] S.-M. Jung and J. Roh, "The linear differential equations with complex constant coefficients and Schrödinger equations," Applied Mathematics Letters, vol. 66, pp. 23-29, 2017.

[5] S.-M. Jung and J. Roh, "Hyers-Ulam stability of the time independent Schrödinger equations," Applied Mathematics Letters, vol. 74, pp. 147-153, 2017.

[6] J. Roh and S.-M. Jung, "Approximation by first-order linear differential equations with an initial condition," Journal of Function Spaces, vol. 2016, Article ID 2406158, 7 pages, 2016.

[7] D. Popa and I. Raşa, "On the Hyers-Ulam stability of the linear differential equation," Journal of Mathematical Analysis and Applications, vol. 381, no. 2, pp. 530-537, 2011.

[8] S. E. Takahasi, T. Miura, and S. Miyajima, "On the Hyers-Ulam stability of the Banach space-valued differential equation $y^{\prime}=$ $\lambda y$, Bulletin of the Korean Mathematical Society, vol. 39, no. 2, pp. 309-315, 2002.

[9] J. Brzdek, D. Popa, I. Rasa, and B. Xu, Ulam Stability of Operators, vol. 1 of Mathematical Analysis and Its Applications, Academic Press, Elsevier, 2018.

[10] E. Gselmann, "Stability properties in some classes of second order partial differential equations," Results in Mathematics, vol. 65, no. 1-2, pp. 95-103, 2014.

[11] B. Hegyi and S.-M. Jung, "On the stability of Laplace's equation," Applied Mathematics Letters, vol. 26, no. 5, pp. 549-552, 2013.

[12] S.-M. Jung, "On the stability of wave equation," Abstract and Applied Analysis, vol. 2013, Article ID 910565, 6 pages, 2013.

[13] S.-M. Jung and K.-S. Lee, "Hyers-Ulam stability of first order linear partial differential equations with constant coefficients," Mathematical Inequalities \& Applications, vol. 10, no. 2, pp. 261266, 2007.

[14] A. Prástaro and T. M. Rassias, "Ulam stability in geometry of PDE's," Nonlinear Functional Analysis and Applications, vol. 8, no. 2, pp. 259-278, 2003.

[15] R. Temam, Navier-Stokes Equations And Nonlinear Functional Analysis, vol. 41 of CBMS-NSF Regional Conference Series in Applied Mathematics, SIAM, Philadelphia, PA, USA, 1983. 


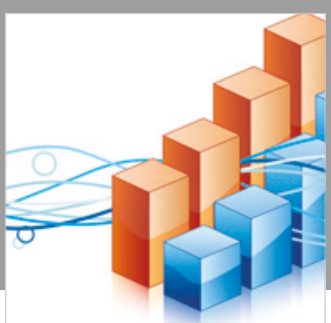

Advances in

Operations Research

\section{-n-m}
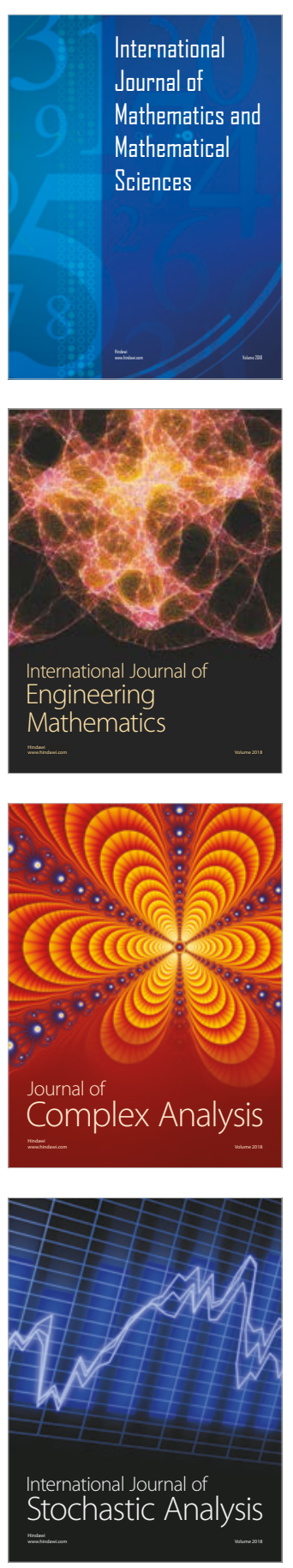
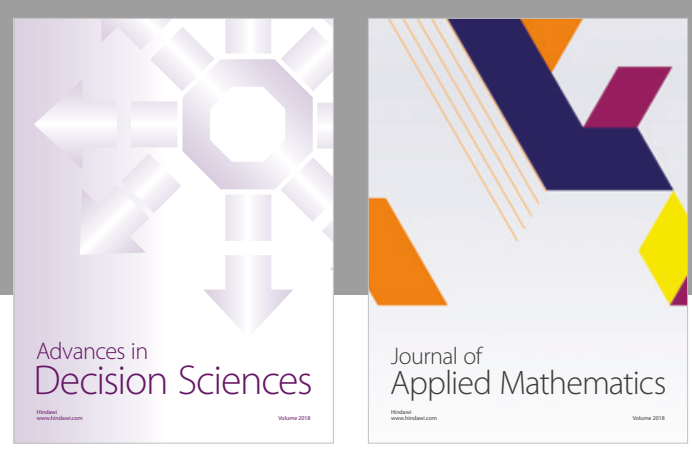

Journal of

Applied Mathematics
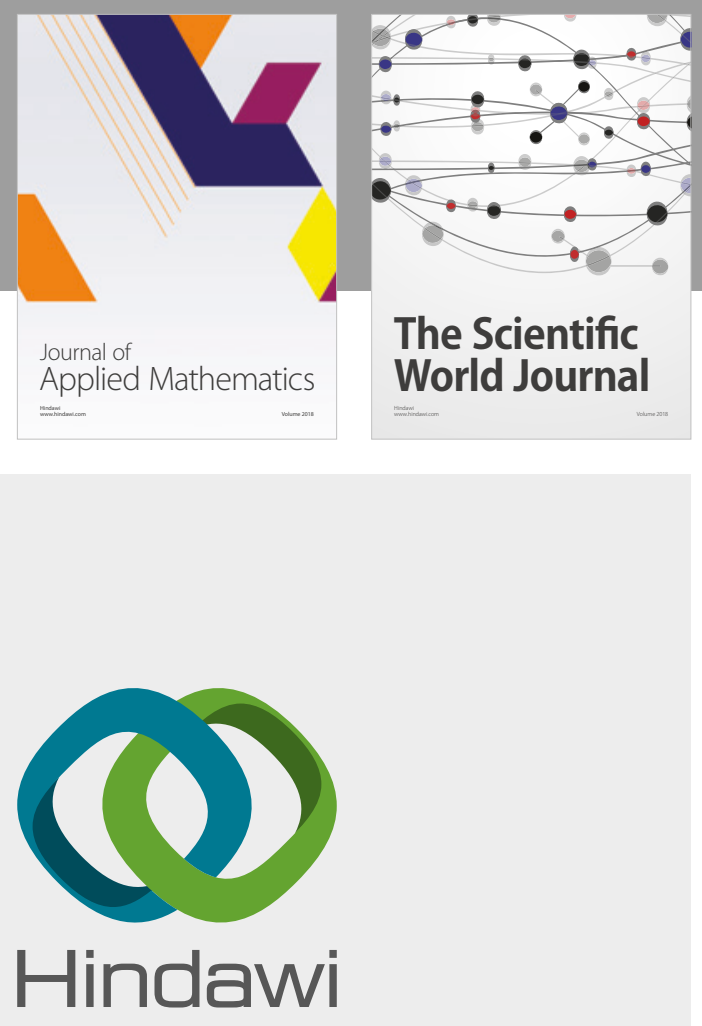

Submit your manuscripts at

www.hindawi.com

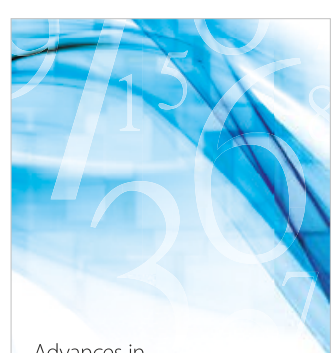

Advances in
Numerical Analysis
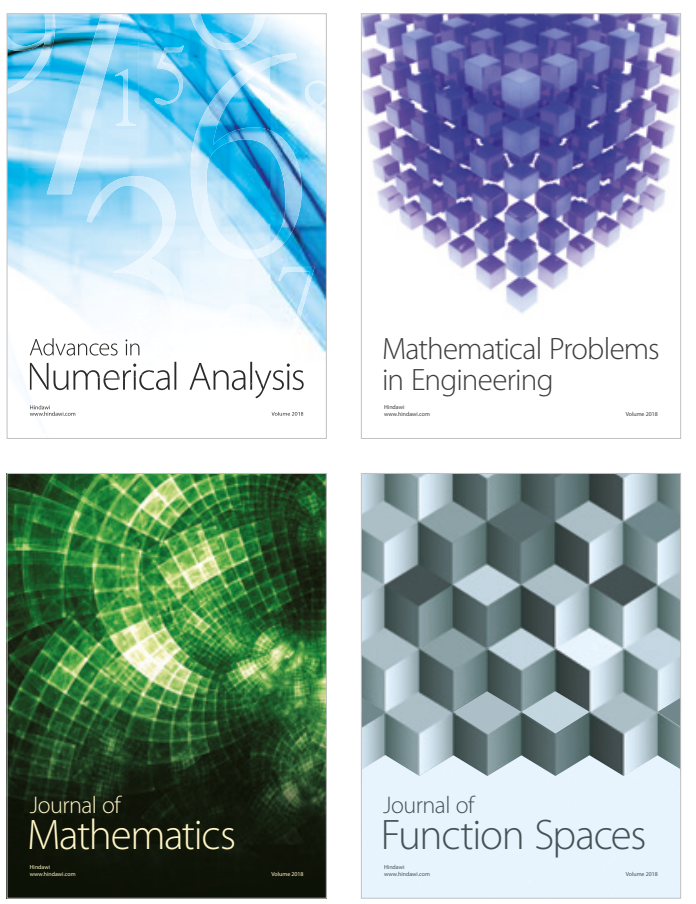

Mathematical Problems in Engineering

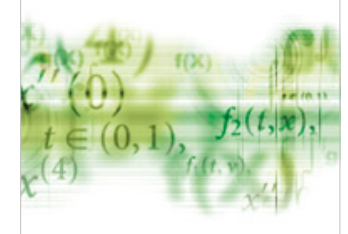

International Journal of

Differential Equations

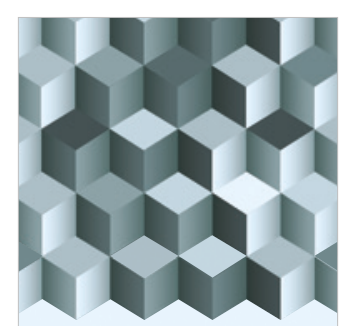

Journal of

Function Spaces

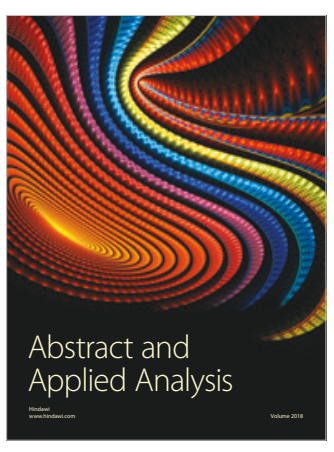

The Scientific

World Journal

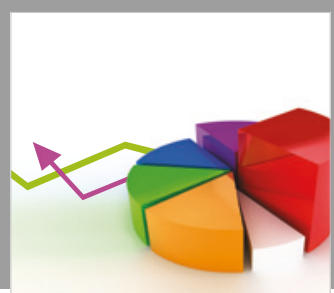

Journal of

Probability and Statistics
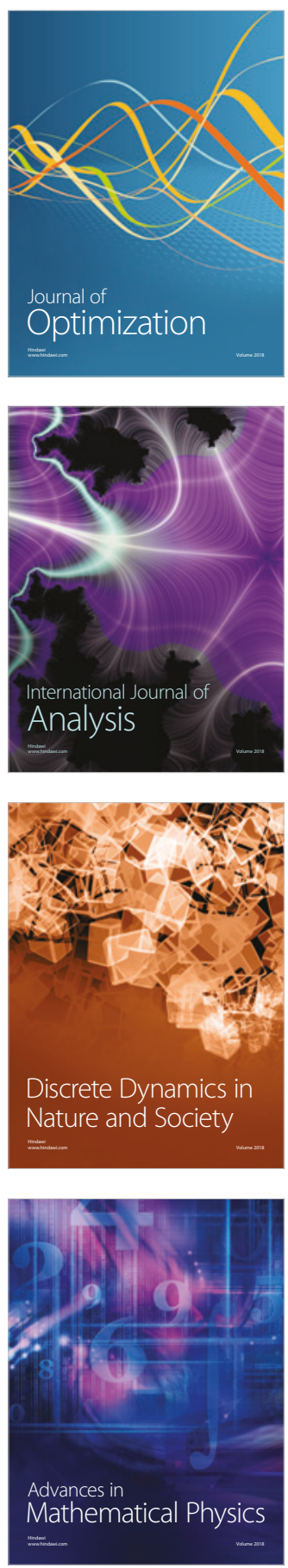